\title{
Non-Canonical Binding of a Small Molecule to Sortilin Alters Cellular Trafficking of ApoB and PCSK9 in Liver Derived Cells
}

\author{
Robert P. Sparks ${ }^{1,7}$, Andres S. Arango ${ }^{3,7}$, Zachary L. Aboff ${ }^{3}$, Jermaine L. Jenkins ${ }^{4}$, \\ Wayne C. Guida ${ }^{5}$, Emad Tajkhorshid ${ }^{1,3,6}$, Charles E. Sparks ${ }^{2}$, Janet D. Sparks ${ }^{2,7}$ and \\ Rutilio A. Fratti ${ }^{1,3, *}$
}

1. Department of Biochemistry, University of Illinois Urbana-Champaign, Urbana, IL 61801

2. Department of Pathology and Laboratory Medicine, University of Rochester Medical Center, Rochester, NY 14642

3. Center for Biophysics and Quantitative Biology, University of Illinois Urbana-Champaign, Urbana, IL 61801

4. Structural Biology \& Biophysics Facility, University of Rochester Medical Center, Rochester, NY 14642

5. Department of Chemistry, University of South Florida, Tampa, FL 33520

6. Beckman Institute for Advanced Science and Technology, University of Illinois at UrbanaChampaign, Urbana, IL 61801

7. These authors contributed equally

*E-mail: rfratti@illinois.edu.

Keywords: Sortilin; Vps10; CPY; Neurotensin; Autophagy; Vps34; phosphatidylinositol 3phosphate; phosphatidylinositol-3,4,5-trisphophate; PCSK9; ApoB containing lipoprotein; LDL receptor; membrane fusion; SNARE; rab7; Ypt7. 


\begin{abstract}
Sortilin regulates hepatic exocytosis and endocytosis of ApoB containing lipoproteins (ApoB-Lp) and mediates the secretion of the subtilase PCSK9. To elucidate connections between these pathways, we previously identified a small molecule (cpd984) that binds to a non-canonical site on Sortilin. In hepatic cells cpd984 augments ApoB-Lp secretion, increases cellular PCSK9 levels, and reduces LDLR expression indicative of reduced secretion of PCSK9. We have shown that insulin-induced ApoB-Lp degradation occurs through Vps34-dependent autophagy. Here we show that the specific Vps34 inhibitor PIK-III enhances ApoB-100 secretion, reducing cellular levels of PCSK9 and Sortilin resulting in reduced LDLR expression, which implicates a role for autophagy in PCSK9 secretion. Results suggest that Sortilin is central to both PCSK9 and ApoB100 secretion. Finally, we found that cpd984 in yeast blocks CPY secretion while increasing vacuolar homotypic fusion in a Vps10-dependent manner, indicating an evolutionarily conserved mechanism required for lysosomal protease trafficking.
\end{abstract}

\title{
INTRODUCTION
}

Trafficking pathways in yeast have been studied extensively as models of eukaryotic biology, which have in turn have been informative for discoveries in mammals related to human disease 1. Vps10 in yeast traffics the lysosomal protease CPY (carboxypeptidase) from the Golgi to the vacuole in an anteretrograde fashion and Vps10 recycles back to the Golgi via the retromer. Vps10 orthologue Sortilin in mammals traffics proteins from the Golgi where it is activated by furin mediated cleavage of its prodomain ${ }^{2}$. Sortilin is responsible for trafficking its ligands between secretory pathways and lysosomal protein pathways ${ }^{3}$. Vps10 and its mammalian counterpart Sortilin release cargo upon entering acidic endosomal compartments, which may be followed by dimerization of the luminal $\beta$-propeller domains of Sortilin. Subsequently, Vps10/Sortilin can be recycled back to the Golgi by the Retromer pathway, which could be mediated by dimerization ${ }^{4}$. In higher eukaryotes, Sortilin is expressed in diverse cell types where it serves as a tissue specific cargo receptor. In neuronal cells, Sortilin participates in the secretion of neurotensin (NT) and other neuromodulators ${ }^{5}$, while in adipose tissue, Sortilin mediates the expression of the type 4 glucose transporter Glut4 at the plasma membrane ${ }^{6}$. In hepatic cells, the Sortilin receptor primarily regulates ApoB containing lipoproteins (Apo-Lp) and the protease PCSK9 (proprotein convertase subtilisin-kexin type 9). When secreted, PCSK9 binds to LDL receptor (LDLR), which targets both LDLR and PCSK9 for lysosomal degradation ${ }^{7}$. 
Through GWAS analysis, SORT1 mutations have been identified as candidates that correlate mutations in Sortilin with cardiovascular disease outcomes ${ }^{8,9}$. Sortilin has complex relationships with lipoprotein metabolism ${ }^{10}$ and in lipid accumulation in arteries ${ }^{11}$. At the plasma membrane, Sortilin is found in Clathrin-coated pits similar to the LDLR, both of which bind ApoB-Lp ${ }^{12}$. Differences in function and ligand specificity for the two receptors is unknown. Further complicating understanding of Sortilin function is its additional role in hepatic VLDL secretion where hepatic knockouts of Sortilin have been described to either increase or decrease VLDL secretion ${ }^{13}$. Sortilin is a multiligand receptor that can interact with the various ligands found on VLDL including $\mathrm{B} 100{ }^{14}, \mathrm{ApoE}^{15,16}$, and $\mathrm{PI}(3,4,5) \mathrm{P}_{3}{ }^{17}$. Circulating VLDL content of $\mathrm{PI}(3,4,5) \mathrm{P}_{3}$ is enriched as compared to HDL and $\mathrm{LDL}^{17}$. The paradox for the ability of Sortilin to both increase or decrease VLDL secretion could relate to the relative concentration and position of ligands on the VLDL surface. Presence of $\mathrm{PI}(3,4,5) \mathrm{P}_{3}$ on lipoproteins relates to insulin signaling where $\mathrm{PI}(3,4,5) \mathrm{P}_{3}$ is considered as a principal mediator of insulin signal transduction, which could provide a mechanism for short-term modulation of VLDL interaction with Sortilin ${ }^{18} 19$.

An additional lipoprotein related ligand for Sortilin is the subtilase PCSK9, which has been shown by SPR to bind to human Sortilin with high affinity ${ }^{20}$. Importantly, this study showed that neurotensin (NT), a natural substrate for Sortilin, did not inhibit PCSK9 binding, even at saturating concentrations ${ }^{21}$. It has been proposed that PCSK9 is chaperoned for secretion by Sortilin. Once PCSK9 is released by sortilin extracellularly, it binds to LDLR resulting in degradation of the associated complex resulting in decreased expression of LDLR on the cell surface as LDLR bound to PCSK9 results in degradation of both PCSK9 and LDLR at the lysosome. We now show that PCSK9 secretion can be modulated by targeting Sortilin. We hypothesize that the relative secretion of Sortilin and PCSK9 can be co-regulated in hepatocytes by VLDL composition and by non-canonical binding of molecules to Site-2 of Sortilin. We suggest that this pathway is a complex and dynamic system for regulating ApoB-100 metabolism, which takes into account the balance of cellular intake (LDLR and VLDLR) and secretion of VLDL, a very low-density progenitor of LDL. We further show that the non-canonical Site-2 of Sortilin. may have evolved from primitive systems for protease trafficking in yeast involving Vps10 with similarities for carboxypeptidase (CPY) and PCSK9 trafficking ${ }^{22}$.

Results presented suggest a potential alternative to current strategies targeting inhibition of the binding of PCSK9 to the LDL receptor (LDLR) ${ }^{23}$. We hypothesize that using Site-2 directed Sortilin binding molecules such as cpd984, hepatic LDLR expression and VLDL secretion could be 
increase under appropriate clinical settings. Furthermore, approaches could be developed to selectively regulate involved pathways by utilizing both sites of Sortilin, as we now show the inverse results of Site- 1 and Site- 2 specific Sortilin molecules ${ }^{24}$.

\section{Results}

\section{Cpd984 and Cpd541 Bind at Different Locations of Human Sortilin}

Our previous studies defined the interaction of cpd984 with Site-2 on Sortilin and its effects on NT binding and VLDL secretion ${ }^{24}$. Here we tested whether cpd984 in combination with cpd541, a Site-1 directed small molecule could be used to examine the relationship between Sites-1 and -2 on Sortilin. In Figure 1A, we show SPR results of a titration of cpd984 concentrations binding to Sortilin. This provides strong evidence that cpd984 binds Sortilin with high affinity, having a $K_{D}$ of $118 \pm 38 \mathrm{nM}$ (Fig. 1A). Next, we determined the binding affinity of Sortilin for cpd541, which we determined has a $K_{D}$ of $6.9 \pm 1.1 \mu \mathrm{M}$ for Sortilin (Fig. 1B). To determine whether binding Site-2 is independent of Site-1, we measured the binding of cpd984 in the presence of a nearly saturating concentration of $10 \mu \mathrm{M} \mathrm{cpd541.} \mathrm{We} \mathrm{found} \mathrm{that} \operatorname{cpd} 984$ bound to Sortilin with a similar affinity in the presence of $10 \mu \mathrm{M}$ cpd541 as in the absence of cpd541 (Fig. 1C). Results are consistent with our previous study showing that cpd984 bound Sortilin in the presence of C-terminal NT ${ }^{17}$.

Using ensemble molecular docking we showed that cpd984 binds to regions of Sortilin comprising Site-2 (Fig. 1D), with a higher percentage than cpd541 (Fig. 1E). Furthermore, we found that the protonated carboxylate on cpd541 did not affect its preferential clustering in Site-1 versus the more hydrophobic Site-2. In parallel, we found that cpd984 with an unprotonated pentaamine ring has a basic $\mathrm{pH}$ of $8.4( \pm 1.2)$, as determined by ab initio calculations using Jaguar ${ }^{25}$. The protonation state of cpd984 had no effect on Site-2 binding, whereas cpd541 protonation state of the carboxylate resulted in a slight enrichment of cpd541 to Site 2 of Sortilin (Fig. 1E). To test whether $\mathrm{pH}$ dependent conformational changes impacted Site-1 or Site-2 binding, using Schrodinger Glide XP, we computationally docked both cpd541 and cpd984 in both Site-1 and Site-2 of a crystal structure of Sortilin at neutral pH (PDB ID: 4PO7) and acidic pH (PDB ID: 6EHO) as shown in Figure 1F. Cpd541 showed dramatically reduced binding to Site-1 of Sortilin as compared to cpd984, indicating that cpd541 is similar to other Site-1 substrates that bind to the NT binding site of Sortilin, and lose affinity at low $\mathrm{pH}^{26}$. Furthermore, docking results confirm that cpd984 shows little discrimination between acidic and neutral pH structure of Sortilin. 
Using molecular dynamics (MD), six 50 ns simulations were run for cpd541 and cpd984 for both Sites-1 and -2 comprising 24 simulations over 1 microsecond in total (Fig. 2A). The three best binding poses of cpd984 to Site-1 were chosen using ensemble poses generated from clusters of Site-1, whereas the three best poses with a salt bridge from cpd541 to Arg292 with the best autodock docking score were chosen and all three poses run in duplicate Figure 1D. For Site-2, the cpd984 pose comprising the closest match to our previously determined pose of cpd984 to Site-2 was used, whereas for cpd541 the three best poses of cpd541 to Site 2 were chosen ${ }^{24}$. Results and analysis of the MD simulations are presented in Figure 2. Cpd984 (Fig. 2A) and cpd541 (Fig. 2D) average distances from Arg292 in Site-1 and Leu539 in Site-2 are given. We found that cpd984 remained within $10 \AA$ from Leu539 throughout the simulation (Fig $2 A){ }^{24}$. In contrast, simulations of cpd984 to Site- 1 showed that it did not stay within $10 \AA$ of R292 for Site1. In addition to the stable association of cpd984 with Site-2, we found that $\operatorname{cpd} 984$ buries itself into the hydrophobic Sortilin beta propeller, between blades 1 and 10. Using F555 as a reference amino acid nearing the end of blade 10 of Sortilin, we show that cpd984 on average over six 50 ns simulations comes closer to this hydrophobic cavity over time (Fig. 2B). A representative endpoint of one of these simulations is presented in a ligand interaction diagram of cpd984 is presented in Figure 2C. Taken together, we hypothesize that the hydrophobic cpd984 is more likely to stay bound to Site-2 than to Site-1. Additionally, as cpd984 does not carry a negative charge at acidic or neutral $\mathrm{pH}$, and has greater association with Site-2 than Site-1 of Sortilin, it is likely that cpd984 binding to Sortilin is $\mathrm{pH}$ independent in the cell (Fig. 1E, F).

Regarding cpd541, we hypothesized that it requires some electrostatic binding to form a salt bridge with Arg292 and stably interact with Site-1 via its carboxylate. In Figure 2D, we found that cpd541 did not stay as tightly associated to Site-2 of Sortilin as compared cpd984. In contrast, cpd541 stably associated with Site- 1 more than Site-2. That said, not all cpd541 simulations remained associated with $\mathrm{R} 292$. A fraction of cpd541 simulations started with a salt bridge to Arg292, eventually stably forming a salt bridge with Lys227 as depicted in Figure 2F depicting one of the final frames of a cpd541 simulation to Site-1 of Sortilin. In Figure 2E, we show the fraction time bound over the course of all 2450 ns simulations as a function of the amount of frames of the simulations as a whole, where either cpd541 or cpd984 stayed within $3.5 \AA$ of a given residue of Sortilin, over the course of all 50,000 frames of the simulation, with each frame representing $1 \mathrm{ps}$. It is clear that cpd984 had a greater fraction bound time than cpd541 in Site-2 of Sortilin, and that cpd541 had a greater fraction time bound than cpd541 to Site-1 (Fig. 2E). Furthermore, the residues of interest for these interactions corresponded well to the NT peptide 
binding modes of Sortilin from PDB ID: 4PO7, which we have hypothesized represents the full NT peptide across the Sortilin cavity connecting Site- 1 and Site-2 of Sortilin ${ }^{24}$.

\section{Sortilin-targeting Small Molecule alters NT Binding and ApoB Secretion}

Using SPR to monitor NT binding to Sortilin we found that cpd541 competed for NT binding to Sortilin in a dose dependent fashion (Fig. 3A). In contrast, we now show that cpd984 enhanced NT binding to Sortilin in a dose dependent fashion ${ }^{24}$. As NT binding has been shown to be mainly guided by Site-1, we hypothesize that cpd541 as an inhibitor most likely binds to NT binding Site1 of Sortilin ${ }^{27}$. Next, we examined the effects of these compounds on ApoB secretion. This was done with different cell lines in which Sortilin levels were differentially reduced with siRNA. Sortilin knockdown cell lines 1-4 expressed $95 \%, 70 \%, 40 \%$ and $10 \%$ of Sortilin, respectively, relative to the scrambled siRNA control cells (Fig. 3B). As previously reported, cpd984 enhanced ApoB secretion in all cell lines, in proportion to the amount of Sortilin expressed (Fig. 3B). In contrast, cpd541 reduced $A$ poB secretion consistent with Site- 1 being the primary binding region of Sortilin for VLDL. When administered together, cpd984 and cpd541 restored VLDL secretion to levels near the untreated control (Fig. 3 C). This suggests that binding Site-2 allosterically regulates Site1 binding.

\section{The Vps34-Specific Inhibitor PIK-III blocks Autophagy and Sortilin Function}

Previous studies of Sortilin indicate a specialized autophagy responsible for insulin-dependent degradation of $A p o B$ in a manner independent of p62 ${ }^{28,29}$. To test effects of Vps34 specific effects on trafficking regulation of VLDL and LDLR we used the Vps34-specific inhibitor PIK-III in lieu of pan-PI 3-Kinase inhibitors such as wortmannin ${ }^{30}$. In order to determine efficacy of PIKIII on autophagy as a control, we measured the autophagy markers LC3-I, LC3-II, and p62 in the presence or absence of wortmannin or PIKIII. Basal autophagic flux was blocked with E/P (E-64d and Pepstatin) ${ }^{31,32}$, or HCQ (hydroxychloroquine) ${ }^{33,34}$, or left untreated. Autophagy activation was detected by presence or absence of LC3-II. We found that LC3-II production was inhibited completely under all three conditions with PIK-III, whereas wortmannin had no effect (Fig. 4A). Furthermore, it appears that neither PIK-III nor wortmannin affected p62 levels under autophagic conditions similar to a previous report suggesting a selective form of autophagy associated with Sortilin ${ }^{28}$. As a measure of insulin-triggered autophagy, we tested whether PIK-III had an effect on the phospho-AKT pathway. We found that PIK-III, unlike wortmannin, failed to inhibit phosphoAKT production (Fig. 4B). 


\section{PIK-III Alters PCSK9 Secretion and LDLR Surface Expression}

After characterizing PIKIII as an autophagy inhibitor, we tested for its effects on Sortilin mediated trafficking by monitoring both VLDL secretion and PCSK9 regulation of LDLR in the presence and absence of wortmannin and PIKIII. Our results indicate that PIK-III affected both VLDL secretion (Fig. 4C) and LDLR expression (Fig. 4D). It appears from Figure 4C that total ApoB increased with PIKIII in both the cell and the media. We hypothesize that as PIKIII inhibits autophagic degradation of $A p o B$, it would then increase the total amount of $A p o B$ available, therefore, we would expect more $A p o B$ shifted towards secretion while the intracellular increase in $A$ poB was due to lack of autophagic degradation. Immunoblotting for intracellular pools of PCSK9, Sortilin, and LDLR showed a decrease in LDLR expression, and reduced levels of Sortilin and PCSK9 (Fig. 4D). We hypothesize that decreased intracellular PCSK9 is consistent with increased PCSK9 secretion, resulting in decreased LDLR expression. The decreased intracellular Sortilin is likely due to the requirement for more Sortilin to be diverted to secretion due to increased ApoB availability.

\section{Effect of cpd541 and cpd984 on the Sortilin-VLDL-PCSK9 Trafficking Pathway.}

We further tested the effect of modulating Sortilin trafficking through defined Site-1 and Site-2 molecules. We found that the Site-2 cpd984 enhanced LDLR expression (Fig. 5A), whereas cpd541 had little effect on PCSK9. We hypothesized that PCSK9 binds to Site-2, which could explain why cpd984 was effective in modulating PCSK9 as opposed to cpd541. Using the proteinprotein docking prediction software ClusPro, we docked PDB ID: 4PO7 Sortilin to PDB ID: 2P4E PCSK9, removing the NT fragments of 4PO7 prior to simulation. All 10 clusters showed PCSK9 binding to Blade-1 of Sortilin (Fig. 5B), and selected the top 3 poses with the lowest overall predicted $\Delta \mathrm{G}$. We ran the three generated clusters for $25 \mathrm{~ns}$ each and analyzed both Sortilin and PCSK9 contact points for binding approximated by having a distance of less than 3.5 Angstroms between the two proteins. This showed that the residues of Sortilin closest to PCSK9 over throughout multiple trajectories remained to be adjacent to Site-2 or on the outside of the Sortilin cavity opposite Site-2. In Figure 5D, we show a root-mean-squared fluctuation (RMSF) analysis of these same trajectories for Sortilin. Residue analysis (Fig. 5C), and RMSF analysis (Fig. 5E) were additionally performed for PCSK9 using residues at its interface with LDLR for reference utilizing a 5 ns MD simulation of PCSK9 to LDLR from PDB ID: 3P5C. All results are consistent with PCSK9 binding to Site-2.

\section{Characterization of VLDL and LDL Sortilin Affinity}


Having concluded previously that $\mathrm{PI}(3,4,5) \mathrm{P}_{3}$ binds to Site- ${ }^{17}$, we tested in vitro binding using SPR to determine the effect of cpd541 and cpd984 on Sortilin binding of $\mathrm{PI}(3,4,5) \mathrm{P}_{3}$. First, we found that diC8-PI $(3,4,5) \mathrm{P}_{3}$ bound with high affinity to crosslinked Sortilin with a $K_{D}$ of $4.2 \mu \mathrm{M} \pm$ $380 \mathrm{nM}$ (Fig. 6A). When we ran the same diC8-PI(3,4,5) $\mathrm{P}_{3}$ concentration curves in the presence of $10 \mu \mathrm{M}$ cpd541 and $10 \mu \mathrm{M}$ cpd984, we found that cpd541 abolished $\mathrm{PI}(3,4,5) \mathrm{P}_{3}$ binding, whereas cpd984 enhanced diC8-PI(3,4,5) $\mathrm{P}_{3}$ binding about 10 -fold to Sortilin with a $K_{D}$ of $474 \mathrm{nM}$ $\pm 85 \mathrm{nM}$. To determine if these effects held true for more native forms of $\mathrm{PI}(3,4,5) \mathrm{P}_{3}$, we used nanodiscs containing $2.5 \% \mathrm{PI}(3,4,5) \mathrm{P}_{3}$. Sortilin bound nanodiscs with an increased binding affinity over diC8-PI(3,4,5) $\mathrm{P}_{3}$ for Sortilin with a $K_{D}$ of $55 \mathrm{nM} \pm 13 \mathrm{nM}$ indicating importance for the lipid membrane to Sortilin binding. Similarly to diC8-PI $(3,4,5) \mathrm{P}_{3}$, the affinity of this interaction was enhanced about 10 -fold by cpd984 to a $K_{D}$ of $5.4 \mathrm{nM} \pm 0.8 \mathrm{nM}$ (Fig. 6B).

We previously showed that circulating VLDL contains more $\mathrm{PI}(3,4,5) \mathrm{P}_{3}$ than LDL ${ }^{17}$. Using these same fractions, we characterized rat derived circulating VLDL and LDL binding to Sortilin using SPR and MST. Here we show that rat VLDL bound Sortilin with a $K_{D}$ of $\sim 4 \mathrm{nM}$, an order of magnitude higher relative to binding LDL with a $K_{D}$ of 54-74 $\mathrm{nM}$. We hypothesize that this was due to differences in particle composition, where VLDL acquired $\mathrm{PI}(3,4,5) \mathrm{P}_{3}$ during the cosynthesis with ApoB. (Fig. 6D, E). These data suggest the differences between LDL and VLDL binding was independent of $A p o B$, as both particle types contain the protein, suggesting a primary difference was the presence of $\mathrm{PI}(3,4,5) \mathrm{P}_{3}$.

\section{Sortilin Small Molecule Effect in Yeast}

The yeast gene VPS10 encodes the type I transmembrane receptor for carboxypeptidase $Y$ (CPY) that cycles between the late Golgi and the prevacuolar compartment ${ }^{22,35}$. The CPY hydrolase enters the ER as an inactive zymogen and is modified by four $\mathrm{N}$-linked polysaccharides forming p1CPY $(67 \mathrm{kDa})^{36}$. Thereafter, p1CPY transits through the Golgi where polysaccharides are extended to form the $\mathrm{p} 2 \mathrm{CPY}$ precursor $(69 \mathrm{kDa})$. p2CPY is transported from late Golgi in vesicles containing the subtilase $\mathrm{Kex} 2$, and is subsequently delivered to the vacuole. Upon arrival, the amino pro-segment of p2CPY is cleaved to yield enzymatically active mature CPY or mCPY (61 kDa). Similar to Sortilin, Vps10 has been shown to have multiple binding sites for different ligands in yeast, however, unlike Sortilin, Vps10 contains two protein sorting domains making Vps10 substantially larger than human Sortilin. ${ }^{37}$ 
We hypothesized that Sortilin trafficking of PCSK9 should share consistencies with Vps10 sorting of CPY, reasoning that the mechanism for regulation of trafficking of proteases destined for the lysosome or vacuole could help elucidate Sortilin cellular itinerary for other ligands such as VLDLApoB. First, we deleted VPS10 from our fusion tester yeast strains DKY6281 and BJ3505 and compared vacuolar levels of CPY and the late endosomal/lysosomal rab GTPase Ypt7. In Figure 7A we show immunoblots of isolated vacuoles from vps10 10 yeast and their parent strains. These results indicate that CPY at the vacuole was reduced in the absence of Vps10 in both strains.

Like PCSK9, we assume that increases in CPY at the vacuole, correspond with decreases in secretion of CPY. We used a classical technique to characterize trafficking defects in response to $\operatorname{cpd} 984^{38}$. To test if a Site-2 homologous area was present on Vps10, we treated wild type yeast cells with cpd984 and measured CPY secretion. We found that cells treated with $20 \mu \mathrm{M}$ cpd984 accumulated substantially more CPY at the yeast vacuole relative to the untreated control

(Fig. 7B). To test whether the increase in vacuolar CPY correlated to a decrease in secreted CPY stores, we treated individual yeast cultures with a concentration curve of cpd984. As shown in Figure 7C, secreted CPY was reduced with increased cpd984 concentrations. Together these data showed that a Site-2-like domain may be present on Vps10.

Finally, we hypothesized that altered trafficking to the vacuole via Vps10 increases in vacuolar CPY due to cpd984 could alter vacuolar homotypic fusion as a measure for increased trafficking to lysosomes. In vitro vacuole fusion assays showed that cpd984 administration resulted in dose dependent increases in fusion of WT vacuoles, while no effect was seen with vps10 $\Delta$ vacuoles, suggesting that proteins responsible for changes in fusogenicity may depend on Vps10 (Fig. 7D). This lends support to a hypothesis that cpd984 enhances Vps34 recruitment resulting in increased trafficking to the lysosome as a potential mechanism.

\section{Discussion}

The endocytic pathway for ApoB-Lp uptake by LDLR in hepatocytes has been studied extensively and forms much of the basis for current therapies controlling high LDL cholesterol in humans ${ }^{39}$. Little is known about the role of Sortilin in this process or how the relative concentrations of these receptors are regulated by Sortilin in this pathway ${ }^{7,10}$. In this study we undertook to further examine the role of Sortilin in LDL/VLDL trafficking. These data show that Sortilin binds VLDL with higher affinity compared to LDL (Fig. 6C, D). We speculate this to be the result of increased 
ligands for Sortilin on the VLDL surface other than $A p o B-100$, e.g. $\mathrm{PI}(3,4,5) \mathrm{P}_{3}$ or $\mathrm{ApoE}$, in contrast to decreased alternative ligands for LDL other than ApoB-100 ${ }^{17,40}$.

Considering that PCSK9 is an additional ligand for Sortilin-mediated secretion in hepatocytes ${ }^{7}$, we further explored the effect of cpd984 on PCSK9 binding to Sortilin. Using functional endpoints in McA cells we demonstrated that cpd984 treated cells retained PCSK9 (Fig. 5A) resulting in increased expression of LDLR. The absence of these effects with the Site-1 ligand cpd541 suggests Site-2 mediates this function. These results are consistent with the hypothesis that Site2 is the primary region of Sortilin for PCSK9 in chaperoning its secretion. Furthermore, we showed that VLDL secretion is inversely modulated by administration of these two compounds (Fig. 3C), that both cpd effects depend on Sortilin expression levels (Fig. 3B), and that when both sites are occupied effects on VLDL secretion are attenuated (Fig. 3C). This indicates the potential for site specific modulation of sortilin chaperone activity.

Regarding NT binding to Sortilin, we showed that cpd541 inhibits NT binding whereas cpd984 enhances binding (Fig. 3A). Results suggest a potential allosteric effect of Site-2 binding on VLDL binding to Site-1, resulting in increased secretion (Fig. 3B, C). Sortilin plays a central role in hepatic ApoB-Lp metabolism, yet its exact role remains enigmatic ${ }^{13}$. The questions that remain are: 1) How do Sortilin knockdowns both increase and decrease hepatic VLDL secretion? ${ }^{10}$; 2) Do separate VLDL ligands interact with one another when binding to Sortilin?; and 3) How important is Sortilin in mediating ApoB-Lp endocytosis through clathrin coated vesicles? Considering the complexity of these questions, we propose a model where Sortilin contains at least two interactive sites for ligand binding.

In addition to mediating ApoB-Lp secretion, Sortilin has a significant role in ApoB degradation by the lysosome ${ }^{41}$. Our previous work showed that blocking autophagy prevents insulin signaling from suppressing $A p o B$ secretion. Whether insulin dependent autophagy is merely an accumulation of basal autophagy is unknown, however insulin-dependent $\mathrm{PI}(3,4,5) \mathrm{P}_{3}$ production its incorporation into VLDL leads us to posit that the production of this lipid diverts ApoB-Lp to the autophagic pathway. We further demonstrated that autophagy regulates PCSK9, Sortilin and LDLR levels (Fig. 4C, D). This process is not dependent on the production of pAKT triggered by insulin, as PIK-III failed to block AKT phosphorylation (Fig. 4B) ${ }^{30}$. The reduction in LDLR, Sortilin and PCSK9 in the presence of PIK-III suggests that loss of autophagy results in increased 
secretion of Sortilin and PCSK9 with resultant PCSK9-mediated reduction in LDLR. Results suggest loss of autophagy affects Sortilin related processes involving both Site-2 and Site-1.

Considering the importance of Site-2 in hepatic ApoB-Lp metabolism, we speculated that the Site2 related pathways for PCSK9 and ApoB-Lp may be conserved in other cell types with some probability that it could have evolved from a primitive pathway present in yeast. The mechanistic role of the yeast CPY protease receptor Vps10, the ancestor of Sortilin, is well well-documented, and also involves at least two ligand binding domains ${ }^{42}$. We used cpd984 to ask whether it might modulate CPY trafficking as it had with Sortilin with respect to the protease PCSK9. Indeed, treating yeast with cpd984 resulted in decreased CPY secretion while increasing its intracellular pool, suggesting CPY sorting is a parallel pathway to PCSK9 sorting as shown by its accumulation at the vacuole and decrease extracellularly (Fig. 7B, C). It appears that these effects are Vps10 dependent as fusion increases with cpd984 were attenuated in vps $10 \Delta$ strains (Fig. 7D).

These studies demonstrate a complex interconnected regulatory system for hepatic lipoprotein metabolism based on evolution of Vps10. Considerable research will be required to completely define this mode of regulation. Considering the complexity of this system, we believe that our observations on Sortilin regulation in hepatocytes and yeast may serve as a paradigm for Sortilin trafficking in other cell types such as neuronal and adipose. Implications of these studies may also have impact on understanding other Sortilin related processes including insulin secretion ${ }^{8,24,43}$, Glut4 transport ${ }^{44-47}$, and plaque formation in Alzheimer's ${ }^{48-50}$ as well as serving as a model for function of other Vps10 homologues such as Vth1, Vth2, and YN94 or Sortilin homologues such as SORCS and SORLA involved in similar processes as Sortilin. Understanding and delineating these protein functions should improve our understanding of the complex regulation of proteins involved in Vps10 family sorting pathways ${ }^{51,52}$.

\section{Methods}

\section{Cell culture, materials and reagents}

McArdle RH-7777 cells (McA cells) were cultured as previously described in serum containing complete Dulbecco's Modified Eagle's Medium (cDMEM) ${ }^{43,53 .}$ To induce insulin sensitivity, McA cells at $50-60 \%$ confluency were incubated for $18 \mathrm{~h}$ in serum-free media consisting of DMEM containing 1\% (w/v) BSA (1\% BSA/DMEM). Human Sortilin (Sortilin) (Ser78-Asn755) with Cterminal 6-His tag was from R\&D Systems, Inc., (Minneapolis, MN). Plasma from fasted Sprague Dawley rats (BioreclamationIVT, Westbury, NY) was used to prepare VLDL ApoB standards. 
Mouse monoclonal antibody to rat B100 was prepared in our laboratory and characterized previously ${ }^{54}$. Rabbit anti-Sortilin antibody was from GeneTex (GTX54854, Irvine, CA), BD Medical (A311709, Franklin Lakes, NJ) or from Abcam (ab16640, Cambridge, MA). Rabbit polyclonal PCSK9 and LDLR antibody was from LSBio (Seattle, WA). Mouse anti-glyceraldehyde phosphate dehydrogenase (GAPDH) from Santa Cruz Biotechnology. Rabbit anti-p62/SQSTM1 was from (Medical \& Biological Laboratories, Nagoya, Japan) and anti-LC3 from (MBL International, Woburn, MA). Lipofectamine 2000, Plus ${ }^{\mathrm{TM}}$ Reagent, and rabbit anti-Vps34 antibody were from Invitrogen/ThermoFisher. Anti-rabbit and anti-mouse horseradish peroxidase (HRP)linked IgG and Hyperfilm ${ }^{\mathrm{TM}}$ were purchased from GE Healthcare. Rabbit anti-p-AKT (9271) and rabbit anti-AKT (9272) were from Cell Signaling Technology (Danvers, MA). Antibody against yeast Ypt7 and actin were previously described ${ }^{55}$, and CPY (10A5B5, ThermoFisher). Mouse anti-pY Platinum 4G10 was from EMD Millipore (Temecula, CA). Horseradish peroxidase linked donkey anti-rabbit IgG (NA9340), sheep anti-mouse IgG (NXA931) and ECL Prime Western Blotting Detection Reagent (RPN2232) were from GE Healthcare. All other materials and reagents were essentially as described previously ${ }^{53}$. diC8-PI(3,4,5) $\mathrm{P}_{3}$ (1,2-dioctanoylphosphatidylinositol 3,4,5-triphosphate) was from Echelon Inc. Wortmannin was from Sigma Chemical Corp and 4'-(cyclopropylmethyl)-N2-4-pyridinyl-[4,5'-bipyrimidine]-2,2'-diamine (PIK-III) from Cayman Chemical (Ann Arbor, MI). Compound 98477898 (2S)-1-methyl-N-3-[(3phenypropanoyl)-amino]phenylpyrrolidine-2-carboxamied (cpd984) and compound 54122218 [2([(1R)-1-phenylethyl]aminocarbonyl)phenyl]amino\}acetic acid were obtained from ChemBridge Corp. (San Diego, CA). Stock solutions of cpd984 and cpd541 (10 mM) were prepared in DMSO, and stored in aliquots at $-20^{\circ} \mathrm{C}$.

\section{Cell Culture}

Rat hepatocytes $(\mathrm{RH})$ were isolated from Sprague-Dawley rat livers, and were cultured on collagen-coated dishes in Waymouth's 751/1 medium containing $0.2 \%(\mathrm{w} / \mathrm{v}) \mathrm{BSA}$ as described previously ${ }^{56}$. Wild-type McA cells were maintained in culture in complete DMEM (cDMEM) ${ }^{53}$. Inhibitors were used at reported concentrations for cpd984 and cpd541. PIK-III was administered at $1 \mu \mathrm{M}$ and wortmannin administered $10 \mu \mathrm{M}$. Inhibitors were validated in $\mathrm{RH}$ where cell toxicity was minimal as determined by LDH release.

\section{Knockdown of Sortilin in McA cells using siRNA}

McA cells were transfected using Fugene6 according to manufacturer's protocol (Promega Corp., Madison WI) using three different pGIPZ based vectors expressing shRNAi targeting rat Sort1 
mRNA (V2LMM_58553, V3LMM_450660, V3LMM_450662), and one scrambled, non-silencing control (GE Healthcare Dharmacon, Lafayette, CO) as previously described ${ }^{24}$. Puromycin selection was performed on McA cells. Sortilin knockdown (KD) from each cell line was examined by immunoblotting.

\section{Immunoblotting}

McA cell lysates were prepared and denatured proteins were separated by SDS-PAGE as described previously ${ }^{43}$. Membranes were incubated with primary antibodies overnight at $4^{\circ} \mathrm{C}$ in blocking buffer with antibody binding detected with species specific secondary HRP-linked antibodies and developed using Amersham ${ }^{\mathrm{TM}}$ Prime reagent (GE Healthcare). Insulin signaling to AKT evaluated with Bio-Rad nitrocellulose membranes with phosphospecific ( $p Y, p$ AKT(Ser473) and mass specific antibodies ${ }^{28}$. Chemiluminescence was measured with ChemiDocXRS+ system (Bio-Rad) and quantified using Image Lab 3.0.1 software from Bio-Rad (Hercules, CA). Autophagy was measured under three conditions: 1. HPQ (hydroxychloroquine) leads to the accumulation of basal LC3-II by blocking fusion between autophagosomes and lysosomes ${ }^{33,34} ; 2$. E/P (E-64d and Pepstatin-A) prevents the fusion of autophagosomes the lysosomes ${ }^{32}$; and 3 . The control were wild type McA.

\section{Immuno-slot blotting}

VLDL and LDL preparations for SPR and MST in Figure 5 were prepared as previously described ${ }^{24}$. Experimental media were adjusted to $1 \%(\mathrm{v} / \mathrm{v})$ protease inhibitor cocktail I (EMD Millipore) and to a salt density of $1.019 \mathrm{~g} / \mathrm{ml}$ by addition of a solution of $\mathrm{NaBr}(\mathrm{d}=1.495 \mathrm{~g} / \mathrm{ml})$. VLDL was isolated by ultracentrifugation in a L-70 Ultracentrifuge (Beckman Coulter, Inc., Fullterton, CA) using a $50 \mathrm{Ti}$ rotor $\left(200,000 \times \mathrm{g}, 18 \mathrm{~h}, 14^{\circ} \mathrm{C}\right)$. Following centrifugation, the top $1.5-2.0 \mathrm{ml} \mathrm{VLDL}$ fraction was removed using a syringe and weighed to determine volume. VLDL samples were applied in triplicate wells $\left(0.2\right.$ to $0.4 \mathrm{ml}$ per well) in a Bio-Dot ${ }^{\circledR}$ SF apparatus (Bio-Rad). Two PVDF membranes were used together for blotting: the top was Immobilon-P (IPVH09120 SF) and the bottom was Immobilon-PSQ (ISEQ09120 SF); both were obtained from EMD Millipore. VLDLApoB standards were prepared from rat plasma VLDL and total ApoB (B100 and B48) and B100 content were determined on stained gels following SDS-PAGE separation ${ }^{57}$. VLDL-ApoB standards in TBS were slotted in duplicate alongside test samples. After filtration, $0.4 \mathrm{ml}$ of TBS was added as a wash. After final filtration, membranes were air dried, rehydrated in methanol and incubated in TBS then in blocking buffer at $4^{\circ} \mathrm{C}$ overnight. At this stage slot blots were evaluated similarly to immunoblotting. After chemiluminescence development and B100 quantitation, slot 
blots were stripped by incubation in Restore ${ }^{\mathrm{TM}}$ PLUS (ThermoFisher) for $15 \mathrm{~min}$ at room temperature and were reblocked overnight at $4^{\circ} \mathrm{C}$. Total ApoB (B100 and B48) present in VLDL was then evaluated following incubation with rabbit polyclonal anti-rat ApoB. The bottom PVDF membrane was carried through the entire procedure to assure there was no "bleed through" of test samples. VLDL-ApoB and VLDL-B100 content were calculated from standard curves generated by VLDL-ApoB standards. Recovery of rat VLDL added to unspent media averaged $94 \% \pm 4.9 \%$ with a CV of $5.2 \%(n=6$ replicates $)$.

\section{Computational modeling and compound screening}

Schrödinger's Maestro program (version 9.3.5) was used as the primary graphical user interface and Maestro version 10.2 (Schrödinger, LLC, New York, NY) was used for ligand interaction diagramming. Virtual screening was performed on compounds contained in ChemBridge libraries (www.chembridge.com) that were prepared with Schrödinger's LigPrep program (Schrödinger, LLC, New York, NY). The virtual screening method was performed using Schrödinger's GLIDE software ${ }^{58}$ on the Sortilin crystal structure PDB ID: 4PO7 27. Compounds were docked on grids generated with Glide with cpd541 docked at a box determined by C-terminal NT and cpd984 docked at a box determined by the N-terminal fragment of NT. Grids were then adapted from alignment of PDB ID:6EHO to PDB ID: 4PO7 and docking performed for all grids using Glide XP setting with results exported into Graphpad Prism.

\section{Simulations of apo and holo Sortilin}

Using the aforementioned crystal structure of Sortilin (PDB ID:4PO7) molecular dynamics simulations were done using NAMD $2.12^{59}$ using the CHARMM36m force field ${ }^{60}$. Prior simulating, the system was prepared using the CHARMM-GUI solution builder, with a salt concentration of $150 \mathrm{mM} \mathrm{NaCl}$. Simulation parameters included constant pressure of 1 atm via Langevin dynamics, as well as a constant temperature of $310 \mathrm{~K}$ using Langevin piston Nosé-Hoover methods ${ }^{61,62}$. Long-range electrostatic forces were evaluated using the particle mesh Ewald (PME) with a $1 \AA$ grid spacing ${ }^{63,64}$. Van der Waals interactions were calculated using a $12 \AA$ cutoff with a force-based switching scheme after $10 \AA$, as well as a 2 fs time step integration via the SETTLE algorithm ${ }^{65}$. Visualization and analysis was done using VMD 1.9.3 ${ }^{66}$. The system was equilibrated for $20 \mathrm{~ns}$ restraining the $\mathrm{Ca}$ atoms of the protein $\left(1.0 \mathrm{kcal} / \mathrm{mol} / \AA^{2}\right)$ to allow for solvation. This was followed by a production run of $50 \mathrm{~ns}$ without restraints for 4 poses taken from ensemble docking, 3 poses with the highest affinity pose for Site-1 of Sortilin run in duplicate for each, and the pose for Site-2 that represented the predicted pose as shown previously ${ }^{17}$. 


\section{Ensemble Molecular Docking of cpd541 and cpd984}

To probe cpd541 and cpd984 interactions with Sortilin, ensemble molecular docking was employed as described ${ }^{67}$. Using snapshots from the $50 \mathrm{~ns}$ production simulation to sample protein dynamics, snapshots were taken every 200 ps. Each of the resultant 250 snapshots were used to dock cpd541 and cpd984 using a $100 \AA$ by $90 \AA$ by $70 \AA \AA$ grid box. Docking was done with an exhaustiveness of 10 , yielding a total of 2500 docked poses. Resultant poses were clustered using a hybrid K-centers/K-medoids algorithm, utilizing an RMSD method ${ }^{68,69}$. Representative poses with highest scoring affinities in clusters closest to Site-1 and Site-2 were selected for further $50 \mathrm{~ns}$ simulations. The resultant drug bound simulations were analyzed with VMD as well as MDAnalysis ${ }^{70,71}$.

\section{Protein-Protein Docking and Simulations}

Characterization of protein-protein interactions between Sortilin (PDB:4PO7) and PCSK9 (PDB:2P4E) was done using the default settings of webserver ClusPro ${ }^{71-73}$. The top three output poses where simulated for a production run of $10 \mathrm{~ns}$ using MD protocols as for Apo and Holo Sortilin. Interacting residue analysis was done using VMD with a $3.5 \AA$ cutoff, over each of the 10 ns trajectories and exported into GraphPad Prism for analysis. Root mean squared fluctuations (RMSF) of $\mathrm{C} \alpha$ atoms of the proteins Sortilin and PCSK9 were analyzed and exported from VMD and plotted using GraphPad Prism.

\section{Microscale Thermophoresis}

Thermophoresis measurements were performed using a Monolith NT.115 labeled thermophoresis instrument ${ }^{72,73}$. Sortilin-His6 was labeled with Ni-NTA Atto-488 according to the manufacturer's protocol as previously described ${ }^{73,74}$. M.O. Control software was used for operation of MST. Target protein concentrations were $50 \mathrm{nM}$ for Sortilin labeled protein. LED excitation power was set to $90 \%$ and MST set to high allowing $3 \mathrm{sec}$ prior to MST on to check for initial fluorescence differences, $25 \mathrm{sec}$ for thermophoresis, and $3 \mathrm{sec}$ for regeneration after MST off. Analysis was performed using M.O. Affinity Analysis Software as the difference between initial fluorescence measure in the first $5 \mathrm{sec}$ as compared with thermophoresis at $15 \mathrm{sec}$. All measurements were performed in PBS buffer $\left(137 \mathrm{mM} \mathrm{NaCl}, 2.7 \mathrm{mM} \mathrm{KCl}, 8 \mathrm{mM} \mathrm{Na}_{2} \mathrm{HPO}_{4}\right.$, and $2 \mathrm{mM} \mathrm{KH} \mathrm{PO}_{4}, \mathrm{pH}$ 7.4) without Tween and binding affinity was generated using Graphpad 
Sigmoidal 4PL fit from points exported from M.O. Affinity Analysis software using $K_{D}$ Model with target concentration fixed at $50 \mathrm{nM}$.

\section{Surface Plasmon Resonance}

Surface plasmon resonance measurements were performed on a Biacore T200 instrument equipped with CM5 sensor chips with $\sim 2000$ response units (RU) of Sortilin covalently immobilized to the surface for VLDL and LDL binding, 3500 RU crosslinked Sortilin for small molecule binding, and a CM5 with $\sim 6500$ RU crosslinked Sortilin for both NT and nanodisc binding. Lipid composition of nanodiscs consisted of $3.023 \mu \mathrm{mol}$ dipalmitoyl phosphatidylcholine (PC), $0.098 \mu \mathrm{mol}$ diC16-PIP3, and $0.78 \mu \mathrm{mol}$ 1-palmitoyl, 2-oleoyl phosphatidylethanolamine $(P E)$, which were prepared as previously described ${ }^{17}$. HBS-DMSO running buffer (10 mM HEPES $\mathrm{pH} 7.4,150 \mathrm{mM} \mathrm{NaCl}, 1 \% \mathrm{DMSO}$ ) was used at a flow rate of $30 \mu \mathrm{l} / \mathrm{min}$ and injections performed with times for association of $90 \mathrm{sec}$, and dissociation of $300 \mathrm{sec}$, followed by injection of buffer to regenerate the Sortilin surface. Regeneration for Ni-NTA non-covalently linked nanodisc experiments utilized EDTA ${ }^{24}$. Regeneration for CM5 NHS/EDC crosslinked Sortilin required a 30 sec injection of $10 \mathrm{mM} \mathrm{NaOH}$ as previously described ${ }^{17}$. Binding was expressed in relative RU; the difference in response between the immobilized protein flow cell and the corresponding control flow cell. NT, cpd984, $5 \% \mathrm{PI}(3,4,5) \mathrm{P}_{3}$ containing nanodiscs and VLDL/LDL were administered to chips containing Sortilin with 1:1 titrations and results exported from BiaEvaluate software into GraphPad prism (GraphPad Software). Cpd984, cpd541 and PI(3,4,5) $\mathrm{P}_{3}$ saturation curves were fit using a specific binding equation with hill slope, whereas all other SPR saturation curves were fit using a 1:1 specific binding model.

\section{Vacuole Isolation and in vitro vacuole fusion assay}

Vacuoles were isolated from BJ3505 ${ }^{75}$ and DKY6281 ${ }^{76}$, which were used for fusion assays by density gradient floatation as previously described ${ }^{72}$. Fusion reactions (30 $\left.\mu \mathrm{L}\right)$ contained $3 \mu \mathrm{g}$

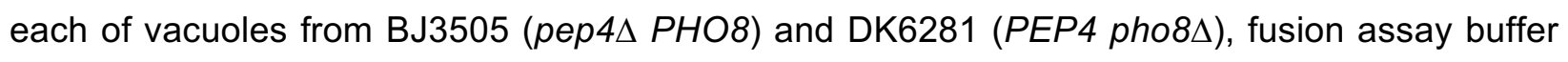
(125 mM KCl, 5 mM MgCl2, 20 mM PIPES-KOH pH 6.8, 200 mM sorbitol), ATP regenerating system ( $1 \mathrm{mM}$ ATP, $29 \mathrm{mM}$ creatine phosphate, $0.1 \mathrm{mg} / \mathrm{ml}$ creatine kinase), $10 \mu \mathrm{M}$ CoA, and 283 $\mathrm{nM}$ Pbi2p and buffer or cpd984. Reactions with or without cpd984 were incubated at $27^{\circ} \mathrm{C}$ for 90 min and the Pho8 activity was measured in $250 \mathrm{mM}$ Tris-Cl PH 8.5, 0.4\% Triton X-100, $10 \mathrm{mM}$ $\mathrm{MgCl}$, $1 \mathrm{mM}$ p-nitrophenyl phosphate. Fusion-dependent alkaline phosphatase maturation was measured by the amount of $p$-nitrophenylate produced. p-Nitrophenylate absorbance was measured at $400 \mathrm{~nm}$. 


\section{Yeast Western Analysis and CPY Secretion}

Vacuoles of BJ3505, DK6281, and their vps10 1 derivatives were isolated and vacuoles were removed by centrifugation $\left(16,000 \times \mathrm{g}, 5 \mathrm{~min}, 4^{\circ} \mathrm{C}\right)$ and SDS sample buffer was added to the supernatants. Samples were heated at $95^{\circ} \mathrm{C}$ for $5 \mathrm{~min}$, resolved by SDS-PAGE, transferred to nitrocellulose, and probed by Western blot in. CPY secretion was measured with vacuoles from BJ3505 and SEY6210 yeast strains incubated with cpd984 were grown at a volume of $1 \mathrm{~L}$ overnight as done for yeast fusion. A $10 \mathrm{~mL}$ sample of YPD media was taken before harvest and spun down to remove cell lysate. TCA precipitation was performed resuspending pellet in approximately $100 \mu \mathrm{L}$ SDS sample buffer, run on SDS-PAGE and blotted for CPY ${ }^{77}$. The remaining $990 \mathrm{~mL}$ of YPD was used for harvesting vacuoles. Vacuoles were measured using Bradford reagent and normalized prior to running SDS-PAGE along with a loading control.

\section{Statistics}

Unless noted, results are expressed as the mean \pm S.E.M., where $n$ equals the number of independent experiments in which replicate analyses were performed in each experiment. Significant differences were assessed using Student's $t$-test with $p$-values $\leq 0.05$ being considered significant.

\section{References}

1. Heitman, J. On the Discovery of TOR As the Target of Rapamycin. PLoS Pathog 11, e1005245 (2015).

2. Nielsen, M. S. et al. The sortilin cytoplasmic tail conveys Golgi-endosome transport and binds the VHS domain of the GGA2 sorting protein. EMBO J 20, 2180-2190 (2001).

3. Staudt, C., Puissant, E. \& Boonen, M. Subcellular Trafficking of Mammalian Lysosomal Proteins: An Extended View. Int J Mol Sci 18, (2016).

4. Itoh, S., Mizuno, K., Aikawa, M. \& Aikawa, E. Dimerization of sortilin regulates its trafficking to extracellular vesicles. J Biol Chem 293, 4532-4544 (2018).

5. Nykjaer, A. \& Willnow, T. E. Sortilin: a receptor to regulate neuronal viability and function. Trends Neurosci 35, 261-270 (2012).

6. Kandror, K. V. The role of sortilin in the "Glut4 Pathway". Commun Integr Biol 11, e1393592 (2018).

7. Gustafsen, C. et al. The hypercholesterolemia-risk gene SORT1 facilitates PCSK9 secretion. Cell Metab 19, 310-318 (2014).

8. Gao, A. et al. Implications of Sortilin in Lipid Metabolism and Lipid Disorder Diseases. DNA Cell Biol 36, 1050-1061 (2017).

9. Musunuru, K. et al. From noncoding variant to phenotype via SORT1 at the $1 \mathrm{p} 13$ cholesterol locus. Nature 466, 714-719 (2010).

10. Strong, A., Patel, K. \& Rader, D. J. Sortilin and lipoprotein metabolism: making sense out 
of complexity. Curr Opin Lipidol 25, 350-357 (2014).

11. Goettsch, C., Kjolby, M. \& Aikawa, E. Sortilin and Its Multiple Roles in Cardiovascular and Metabolic Diseases. Arterioscler Thromb Vasc Biol 38, 19-25 (2018).

12. Martínez-Oliván, J., Arias-Moreno, X., Velazquez-Campoy, A., Millet, O. \& Sancho, J. LDL receptor/lipoprotein recognition: endosomal weakening of $A p o B$ and $A p o E$ binding to the convex face of the LR5 repeat. FEBS J 281, 1534-1546 (2014).

13. Sparks, C. E., Sparks, R. P. \& Sparks, J. D. The enigmatic role of sortilin in lipoprotein metabolism. Curr Opin Lipidol 26, 598-600 (2015).

14. Strong, A. et al. Hepatic sortilin regulates both apolipoprotein B secretion and LDL catabolism. J Clin Invest 122, 2807-2816 (2012).

15. Carlo, A. S. Sortilin, a novel APOE receptor implicated in Alzheimer disease. Prion 7, 378382 (2013).

16. Carlo, A. S. et al. The pro-neurotrophin receptor sortilin is a major neuronal apolipoprotein $E$ receptor for catabolism of amyloid- $\beta$ peptide in the brain. $J$ Neurosci 33, 358-370 (2013).

17. Sparks, R. P. et al. Phosphatidylinositol $(3,4,5)$-trisphosphate binds to sortilin and competes with neurotensin: Implications for very low density lipoprotein binding. Biochem Biophys Res Commun 479, 551-556 (2016).

18. Ijuin, T. \& Takenawa, T. Regulation of insulin signaling by the phosphatidylinositol 3,4,5triphosphate phosphatase SKIP through the scaffolding function of Pak1. Mol Cell Biol 32 , 3570-3584 (2012).

19. Haas, M. E., Attie, A. D. \& Biddinger, S. B. The regulation of ApoB metabolism by insulin. Trends Endocrinol Metab 24, 391-397 (2013).

20. Kjolby, M., Nielsen, M. S. \& Petersen, C. M. Sortilin, encoded by the cardiovascular risk gene SORT1, and its suggested functions in cardiovascular disease. Curr Atheroscler Rep 17, 496 (2015).

21. Mazella, J. et al. The 100-kDa neurotensin receptor is gp95/sortilin, a non-G-proteincoupled receptor. J Biol Chem 273, 26273-26276 (1998).

22. Marcusson, E. G., Horazdovsky, B. F., Cereghino, J. L., Gharakhanian, E. \& Emr, S. D. The sorting receptor for yeast vacuolar carboxypeptidase $Y$ is encoded by the VPS10 gene. Cell 77, 579-586 (1994).

23. Nozue, T. Lipid Lowering Therapy and Circulating PCSK9 Concentration. J Atheroscler Thromb 24, 895-907 (2017).

24. Sparks, R. P. et al. Sortilin facilitates VLDL-B100 secretion by insulin sensitive McArdle RH7777 cells. Biochem Biophys Res Commun 478, 546-552 (2016).

25. Bochevarov, A. D. et al. Jaguar: A high-performance quantum chemistry software program with strengths in life and materials sciences. Int. J. Quanum Chem 113, 2110-2142 (2013).

26. Leloup, N. et al. Low $\mathrm{pH}$-induced conformational change and dimerization of sortilin triggers endocytosed ligand release. Nat Commun 8, 1708 (2017).

27. Quistgaard, E. M. et al. Revisiting the structure of the Vps10 domain of human sortilin and its interaction with neurotensin. Protein Sci 23, 1291-1300 (2014).

28. Sparks, J. D., O’Dell, C., Chamberlain, J. M. \& Sparks, C. E. Insulin-dependent apolipoprotein B degradation is mediated by autophagy and involves class I and class III phosphatidylinositide 3-kinases. Biochem Biophys Res Commun 435, 616-620 (2013).

29. Amengual, J. et al. Autophagy Is Required for Sortilin-Mediated Degradation of Apolipoprotein B100. Circ Res 122, 568-582 (2018).

30. Dowdle, W. E. et al. Selective VPS34 inhibitor blocks autophagy and uncovers a role for NCOA4 in ferritin degradation and iron homeostasis in vivo. Nat Cell Biol 16, 1069-1079 (2014).

31. Dornhof, R. et al. Stress fibers, autophagy and necrosis by persistent exposure to PM2.5 from biomass combustion. PLoS One 12, e0180291 (2017).

32. Wu, J. et al. Autophagy protects against cholesterol-induced apoptosis in pancreatic $\beta$ - 
cells. Biochem Biophys Res Commun 482, 678-685 (2017).

33. Nordstrøm, L. U. et al. Discovery of autophagy inhibitors with antiproliferative activity in lung and pancreatic cancer cells. ACS Med Chem Lett 6, 134-139 (2015).

34. Parks, A. \& Marceau, F. Lysosomotropic cationic drugs induce cytostatic and cytotoxic effects: Role of liposolubility and autophagic flux and antagonism by cholesterol ablation. Toxicol Appl Pharmacol 305, 55-65 (2016).

35. Ronan, B. et al. A highly potent and selective Vps34 inhibitor alters vesicle trafficking and autophagy. Nat Chem Biol 10, 1013-1019 (2014).

36. Vida, T. A., Huyer, G. \& Emr, S. D. Yeast vacuolar proenzymes are sorted in the late Golgi complex and transported to the vacuole via a prevacuolar endosome-like compartment. $J$ Cell Biol 121, 1245-1256 (1993).

37. Fitzgerald, I. \& Glick, B. S. Secretion of a foreign protein from budding yeasts is enhanced by cotranslational translocation and by suppression of vacuolar targeting. Microb Cell Fact 13, 125 (2014).

38. Wolf, D. H. \& Schäfer, A. CPY* and the power of yeast genetics in the elucidation of quality control and associated protein degradation of the endoplasmic reticulum. Curr Top Microbiol Immunol 300, 41-56 (2005).

39. Nair, P. Brown and Goldstein: the cholesterol chronicles. Proc Natl Acad Sci U S A 110, 14829-14832 (2013).

40. Mamotte, C. D., Sturm, M., Foo, J. I., van Bockxmeer, F. M. \& Taylor, R. R. Comparison of the LDL-receptor binding of VLDL and LDL from apoE4 and apoE3 homozygotes. Am J Physiol 276, E553-7 (1999).

41. Sparks, J. D., Phung, T. L., Bolognino, M. \& Sparks, C. E. Insulin-mediated inhibition of apolipoprotein $B$ secretion requires an intracellular trafficking event and phosphatidylinositol 3-kinase activation: studies with brefeldin A and wortmannin in primary cultures of rat hepatocytes. Biochem J 313, 567-574 (1996).

42. Jørgensen, M. U., Emr, S. D. \& Winther, J. R. Ligand recognition and domain structure of Vps10p, a vacuolar protein sorting receptor in Saccharomyces cerevisiae. Eur J Biochem 260, 461-469 (1999).

43. Sparks, J. D., Magra, A. L., Chamberlain, J. M., O’Dell, C. \& Sparks, C. E. Insulin dependent apolipoprotein $B$ degradation and phosphatidylinositide 3-kinase activation with microsomal translocation are restored in McArdle RH7777 cells following serum deprivation. Biochem Biophys Res Commun 469, 326-331 (2016).

44. Kioumourtzoglou, D., Pryor, P. R., Gould, G. W. \& Bryant, N. J. Alternative routes to the cell surface underpin insulin-regulated membrane trafficking of GLUT4. J Cell Sci 128, 2423-2429 (2015).

45. Morris, N. J. et al. Sortilin is the major 110-kDa protein in GLUT4 vesicles from adipocytes. J Biol Chem 273, 3582-3587 (1998).

46. Pan, X., Zaarur, N., Singh, M., Morin, P. \& Kandror, K. V. Sortilin and retromer mediate retrograde transport of Glut4 in 3T3-L1 adipocytes. Mol Biol Cell 28, 1667-1675 (2017).

47. Shi, J. \& Kandror, K. V. Sortilin is essential and sufficient for the formation of Glut4 storage vesicles in 3T3-L1 adipocytes. Dev Cell 9, 99-108 (2005).

48. Capsoni, S. et al. SorLA deficiency dissects amyloid pathology from tau and cholinergic neurodegeneration in a mouse model of Alzheimer's disease. J Alzheimers Dis 33, 357371 (2013).

49. Ruan, C. S. et al. Sortilin inhibits amyloid pathology by regulating non-specific degradation of APP. Exp Neurol 299, 75-85 (2018).

50. Zhao, Y., Cui, J. G. \& Lukiw, W. J. Reduction of sortilin-1 in Alzheimer hippocampus and in cytokine-stressed human brain cells. Neuroreport 18, 1187-1191 (2007).

51. Leloup, N., Chataigner, L. M. P. \& Janssen, B. J. C. Structural insights into SorCS2-Nerve Growth Factor complex formation. Nat Commun 9, 2979 (2018). 
52. Nielsen, M. S. et al. Different motifs regulate trafficking of SorCS1 isoforms. Traffic 9, 980994 (2008).

53. Chamberlain, J. M., O'Dell, C., Sparks, C. E. \& Sparks, J. D. Insulin suppression of apolipoprotein B in McArdle RH7777 cells involves increased sortilin 1 interaction and lysosomal targeting. Biochem Biophys Res Commun 430, 66-71 (2013).

54. Sparks, J. D., Bolognino, M., Trax, P. A. \& Sparks, C. E. The production and utility of monoclonal antibodies to rat apolipoprotein B lipoproteins. Atherosclerosis 61, 205-211 (1986).

55. Miner, G. E., Starr, M. L., Hurst, L. R. \& Fratti, R. A. Deleting the DAG kinase Dgk1 augments yeast vacuole fusion through increased Ypt7 activity and altered membrane fluidity. Traffic 18, 315-329 (2017).

56. Sparks, J. D. et al. Acute suppression of apo B secretion by insulin occurs independently of MTP. Biochem Biophys Res Commun 406, 252-256 (2011).

57. Chirieac, D. V., Davidson, N. O., Sparks, C. E. \& Sparks, J. D. PI3-kinase activity modulates apo B available for hepatic VLDL production in apobec-1-/- mice. Am J Physiol Gastrointest Liver Physiol 291, G382-8 (2006).

58. Halgren, T. A. et al. Glide: a new approach for rapid, accurate docking and scoring. 2. Enrichment factors in database screening. J Med Chem 47, 1750-1759 (2004).

59. Phillips, J. C. et al. Scalable molecular dynamics with NAMD. J Comput Chem 26, 1781$1802(2005)$.

60. Huang, J. et al. CHARMM36m: an improved force field for folded and intrinsically disordered proteins. Nat Methods 14, 71-73 (2017).

61. Feller, S. E., Zhang, Y., Pastor, R. W. \& Brooks, B. R. Constant Pressure Molecular Dynamics Simulation: The Langevin Piston Method. J Chem Phys 103, 4613-4621 (1995).

62. Martyna, G. J., Tobias, D. J. \& Klein, M. L. Constant Pressure Molecular Dynamics Algorithms. J Chem Phys 101, 4177-4189 (1994).

63. Darden, T., York, D. \& Pedersen, L. G. Particle Mesh Ewald: An N·log(N) Method for Ewald Sums in Large Systems. J Chem Phys 98, 10089-10092 (1993).

64. Essmann, U. et al. A Smooth Particle Mesh Ewald: An $N \cdot \log (\mathrm{N})$ Method for Ewald Sums in Large Systems. J Chem Phys 103, 8577-8593 (1995).

65. Miyamoto, S. \& Kollman, P. A. SETTLE: An Analytical Version of the SHAKE and RATTLE Algorithm for Rigid Water Molecules. J Comput Chem 13, 952-962 (1992).

66. Humphrey, W., Dalke, A. \& Schulten, K. VMD - Visual Molecular Dynamics. J Mol Graphics 14, 22-28 (1996).

67. Trott, O. \& Olson, A. J. AutoDock Vina: improving the speed and accuracy of docking with a new scoring function, efficient optimization, and multithreading. J Comput Chem 31, 455461 (2010).

68. Beauchamp, K. A. et al. MSMBuilder2: Modeling Conformational Dynamics at the Picosecond to Millisecond Scale. J Chem Theory Comput 7, 3412-3419 (2011).

69. Pande, V. S., Beauchamp, K. \& Bowman, G. R. Everything you wanted to know about Markov State Models but were afraid to ask. Methods 52, 99-105 (2010).

70. Gowers, R. J. et al. MDAnalysis: A Python package for the rapid analysis of molecular dynamics simulations. Proceedings of the 15th Python in Science Conference doi:10.25080/majora-629e541a-00e, 98-105 (2016).

71. Michaud-Agrawal, N., Denning, E. J., Woolf, T. B. \& Beckstein, O. MDAnalysis: a toolkit for the analysis of molecular dynamics simulations. J Comput Chem 32, 2319-2327 (2011).

72. Sparks, R. P. et al. A small-molecule competitive inhibitor of phosphatidic acid binding by the AAA+ protein NSF/Sec18 blocks the SNARE-priming stage of vacuole fusion. J Biol Chem (2019).

73. Starr, M. L. et al. Phosphatidic acid induces conformational changes in Sec18 protomers that prevent SNARE priming. J Biol Chem 294, 3100-3116 (2019). 
74. Sparks, R. P. \& Fratti, R. Use of Microscale Thermophoresis (MST) to Measure Binding Affinities of Components of the Fusion Machinery. Methods Mol Biol 1860, 191-198 (2019).

75. Jones, E. W., Zubenko, G. S. \& Parker, R. R. PEP4 gene function is required for expression of several vacuolar hydrolases in Saccharomyces cerevisiae. Genetics 102, 665-677 (1982).

76. Haas, A., Conradt, B. \& Wickner, W. G-protein ligands inhibit in vitro reactions of vacuole inheritance. J Cell Biol 126, 87-97. (1994).

77. Link, A. J. \& LaBaer, J. Trichloroacetic acid (TCA) precipitation of proteins. Cold Spring Harb Protoc 2011, 993-994 (2011).

\section{Acknowledgements}

This paper is dedicated to Janet DeHoff Sparks the wife of Charles Edward Sparks and mother of Robert Pleasants Sparks. Following training at the University of Pennsylvania and PostDoctoral training at Wistar, Janet went on to become a Full Professor at University of Rochester Medical Center in the Department of Pathology. Janet loved to get her work done early in the morning, go to Midtown Athletic Center, and then go right back to work to perform experiments. Janet continued this routine until her death in Sarasota, FI from an unexpected heart attack that may have been due to an as yet well characterized genetic lipoprotein disorder. Many of the experiments in this paper were performed by Janet herself, not a graduate student. Furthermore, many of the experiments were also performed by Janet in the presence of her son Robert, who hung on to her proverbial coat tails attempting to get access to the latest data while performing minimal manual labor.

This research was supported by grants from the National Institutes of Health (R01-GM101132 to RAF, and P41-GM104601, U01-GM111251 and U54-GM087519 to ET), the National Science Foundation (MCB 1818310 to RAF), and the Office of Naval Research (ONR N00014-16-1-2535 to E.T.). Computational resources were provided by XSEDE (XSEDE MCA06N060) and Blue Waters (ACl-1440026). SPR was aided by the help of Dr. Jermaine Jenkins at the University of Rochester Structural Biology \& Biophysics Facility with support from NIH NCRR grant 1 S10 RR027241, as well as NIH NIAID P30AI078498 and the University of Rochester School of Medicine and Dentistry.

\section{Author Contributions}

RPS, JDS, ASA, CES and RAF conceived the project and designed experiments. RPS, JDS, ASA, ZLA, and JLK performed the experiments and analyzed data. JDS, CES and RAF 
supervised the research. RPS, ASA, JDS, CES and RAF wrote the manuscript with input from all authors.

\section{Competing interests}

The authors declare no competing interests.

\section{Data availability}

All data generated or analyzed during this study are included in this published article.

The abbreviations used are: ApoB-Lp, $A p o B$ containing lipoproteins; $\mathrm{PI}(3,4,5) \mathrm{P}_{3}$, phosphatidylinositol $(3,4,5)$-trisphosphate; Sortilin, human Sortilin; NT, neurotensin; CtermNT, Cterminal neurotensin; NtermNTC, N-terminal neurotensin modified from carboxyl to amide Cterminal; MST, microscale thermophoresis; VLDL, very low density lipoprotein; B100, apolipoprotein B derived from unedited $A p o b$ mRNA; SPR, surface plasmon resonance; CPY, carboxypeptidase Y; McA, McCardle cells. 

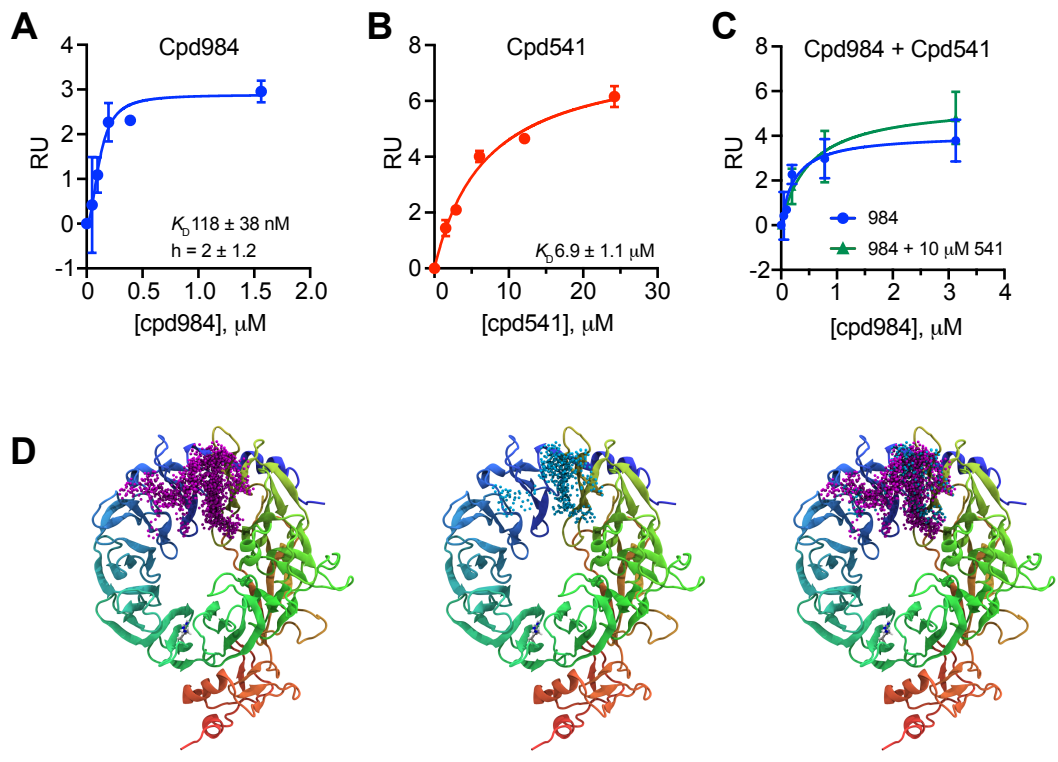

cpd984 at Site-2

cpd541 at Site-2

cpd541 \& cpd 984

at Site-2
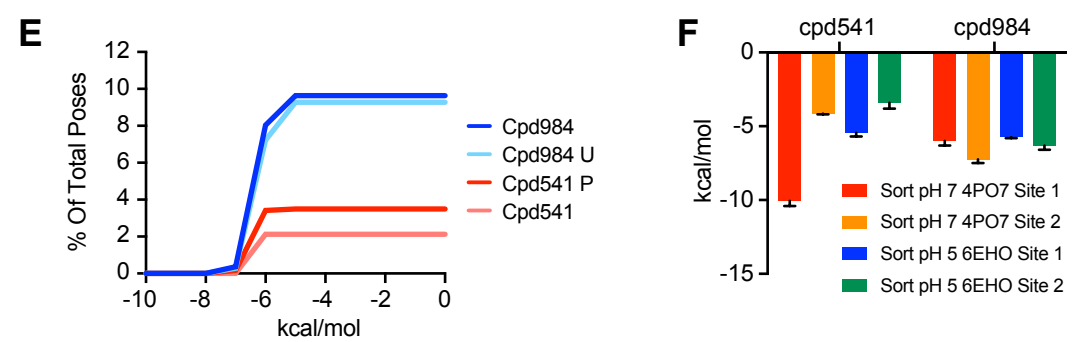

Figure 1. Experimental and Computational Characterization of cpd541 and cpd984. A, SPR determined binding affinity of cpd984 to on CM5 chip loaded with $\sim 3000 \mathrm{RU}$ of human Sortilin with saturation curve (blue) indicating $K_{D} 118 \pm 38 \mathrm{nM}$. B, SPR as in 1a, of cpd541 to Sortilin with saturation curve (red) $K_{D} 6.9 \pm 1.1 \mu \mathrm{M}$. C, SPR as in $1 \mathrm{a}$ and $1 \mathrm{~b}$ with cpd984 titration in the presence (green) and absence (blue) of $10 \mu \mathrm{M}$ cpd541 with saturation curves. D, Resultant poses from ensemble docking for cpd984 (purple) and cpd541 (cyan) at Site-2 of Sortilin alone and superimposed. e, Percent of overall poses from ensemble docking versus relative docking score for cpd984 (red) and cpd541 (blue) where the $Y$ axis is the percent of all poses for ensemble docking that bind site 2 of Sortilin, with cpd984 having about $9 \%$ percent of all poses in site 2 and cpd541 having about 3 percent of all poses. f, Schrodinger Glide Xp scores of cpd541 and cpd984 docked in site 1 and site 2 of $\sim p H 7$ PDB ID: 4PO7 and pH5.5 PDB ID: 6EHO. 


$$
\text { A }
$$

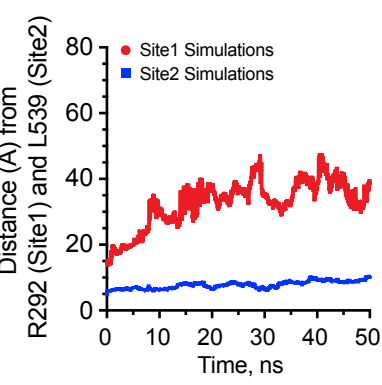

C

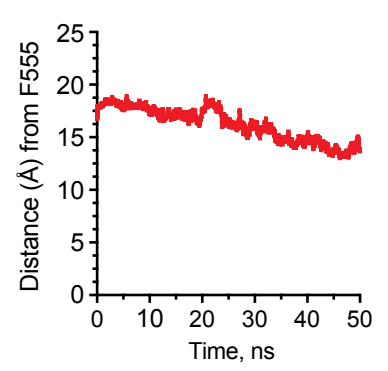

D

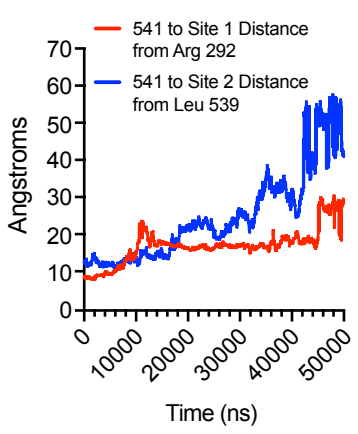

E

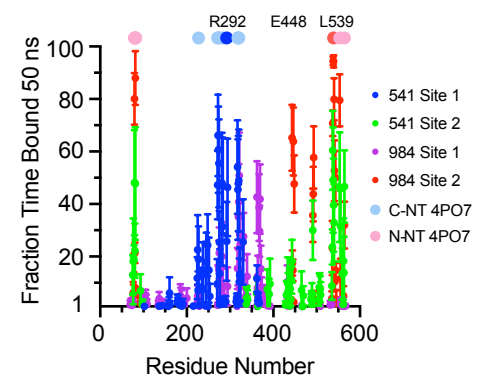

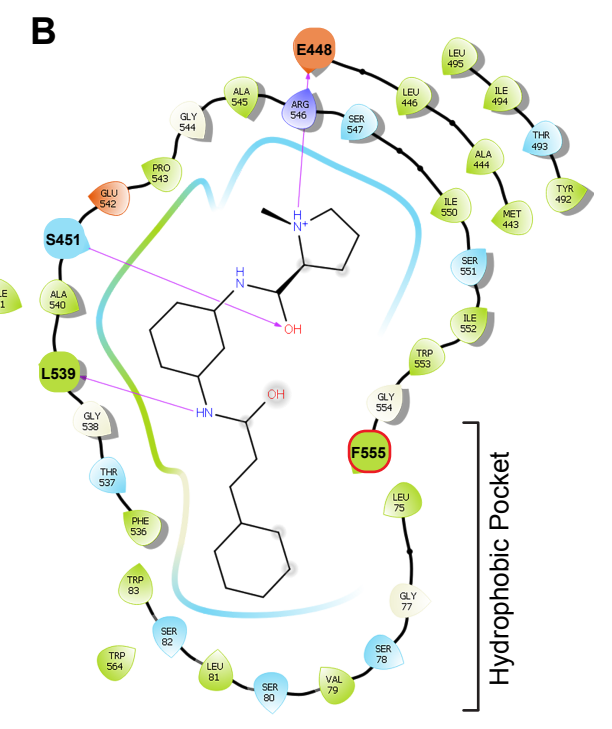

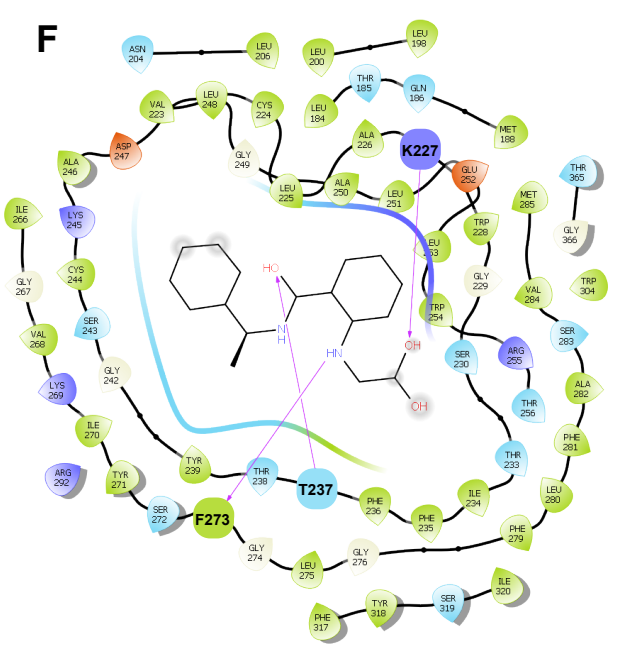

Figure 2. Computational Characterization of cpd984 to Sortilin Site-1 and Site-2. A, Simulations of bound cpd984 poses taken from ensemble docking as in Figure 1d, 1e with Site-2 pose taken from closest ensemble docking pose to pose $(n=6)$ and the three highest scoring poses for cpd984 to Site-1 ( $\mathrm{n}=2$ each) based on ensemble docking. Center of mass difference over time (50 ns) between cpd984 and representative residue of Site-1 R292 for the three duplicate simulations of cpd984 to Site-1 are shown in red, whereas center of mass differences 
between cpd984 and representative residue of Site-2 Leu539 for the six duplicate simulations of cpd984 to Site-2 are shown in blue. B, Ligand interaction diagram taken from a representative simulation from Figure $2 a$ of cpd984 to Site-2 of Sortilin at the end of the 50 ns MD simulation. C, Center of mass difference over time as in Figure 2a between cpd984 and a representative residue of the hydrophobic ligand binding pocket of Sortilin adjacent to Site-2 Phe555 of six simulations of Site-2 bound ligands). D, Simulations of bound cpd541 as in Fig. 2A, with center of mass taken from Arg202 and Leu539 as in Fig. 2A, from top 3 cpd541 ensemble docking poses in site 2 ( $n=2)$ and cpd541 poses from site 1 with a salt bridge between cpd541 and Arg292. e, Residue analysis for all 2450 ns simulations of cpd984 and cpd541 in site 1 and site 2 of Sortilin with fraction time bound calculated by determining number of frames (5000) where a residue of Sortilin was within $3.5 \AA$ of either cpd984 or cpd541. C-term and N-term NT used as a legend at $100 \%$ over the one frame taken from PDB coordinates of PDB ID: 4PO7. F, Ligand interaction diagram taken from a representative simulation from Figure $2 d$ of cpd541 to Site- 1 of Sortilin at the end of the 50 ns MD simulation. 

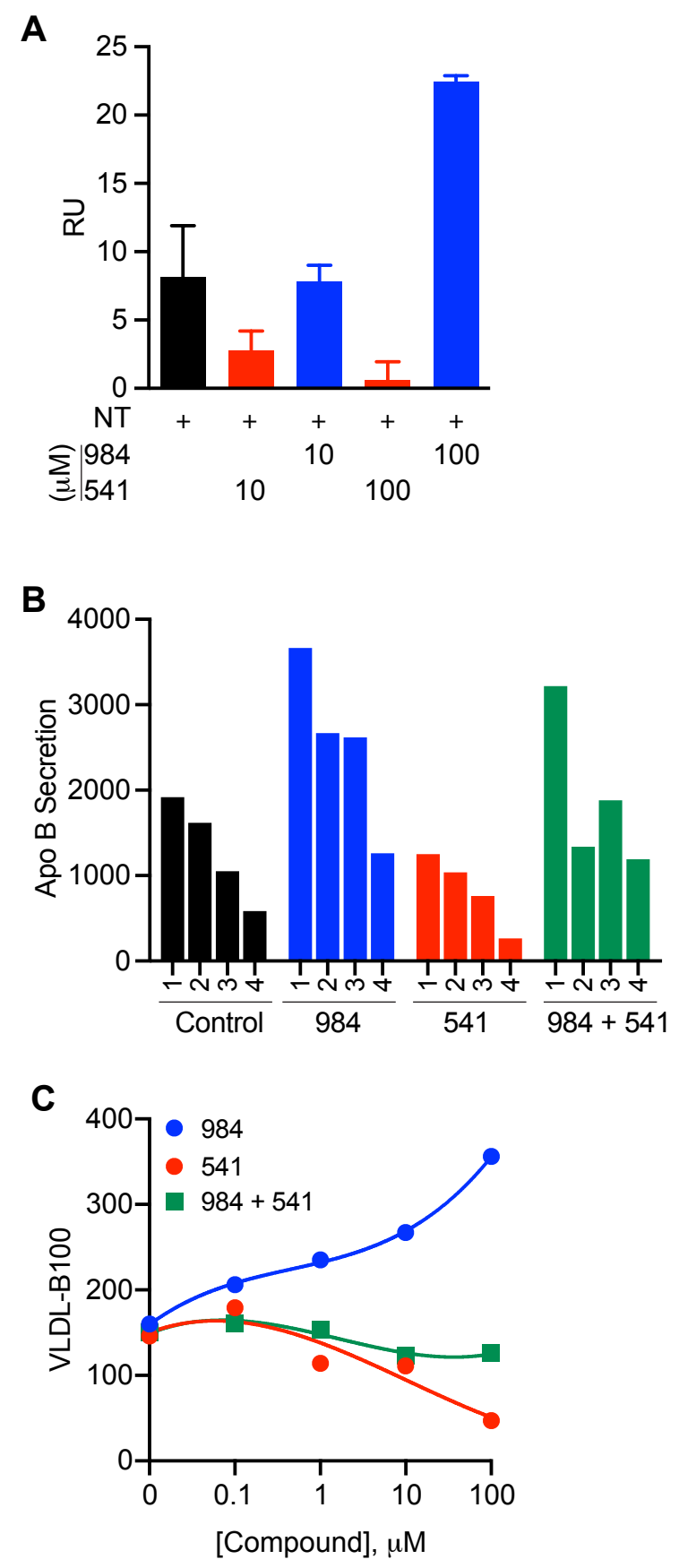

Figure 3. Cpd984/cpd541 Differential Effects on NT Binding and ApoB Secretion ion McA Cells. A, SPR analysis following administration of $100 \mathrm{nM} \mathrm{NT}$ alone and in the presence of either cpd984 or cpd541 at concentrations of 10 and $100 \mu \mathrm{M}$. RU subtractions of cpd984 and cpd541 alone were performed for injections at the corresponding concentrations in the presence of neurotensin in order to depict the effect of these compounds on the binding of neurotensin to 
Sortilin. B, McA knockdowns with varying levels of Sortilin were measured for secretion of VLDLB100 into media and assessed by immuno-slot blot. The effect of Sortilin $K_{D}$ on VLDL-B100 secretion and VLDL-ApoB secretion by insulin sensitive McA cells (1\% BSA/DMEM). Results are the average of triplicate plates for each condition \pm S.D. * $p<0.05$. C, Insulin sensitive McA were incubated with increasing concentrations of cpd541 or cpd984 and secretion of VLDL-B100 into media was assessed by immune-slot blotting. 
A

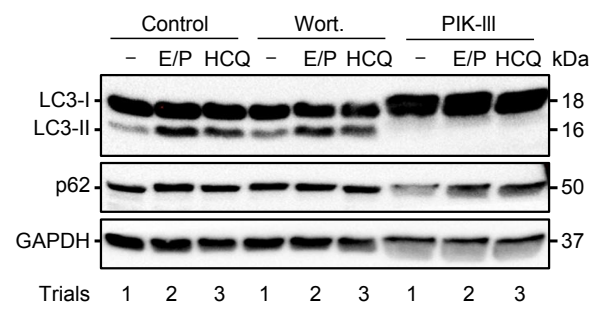

B
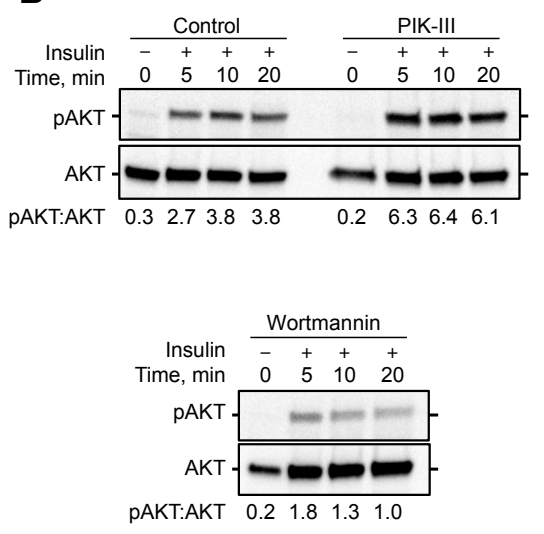

C
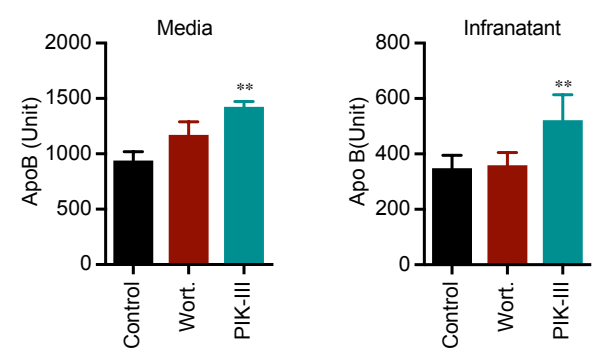

D

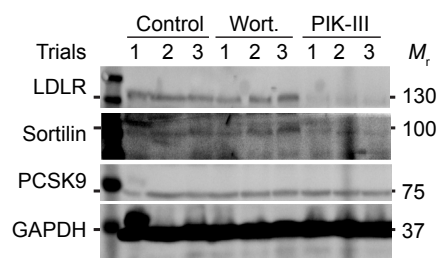

E

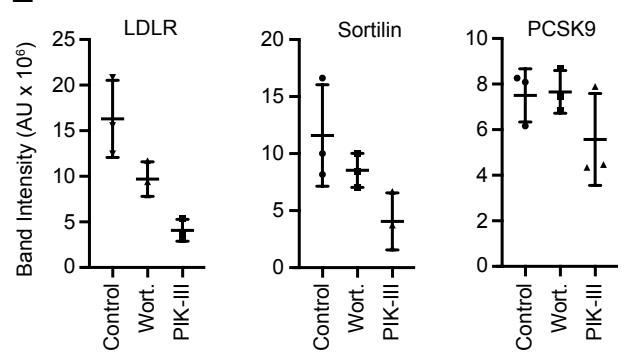

Figure 4. PIK-III Increases Secretion of Both ApoB and PCSK9. A, Measurement of autophagic protein expression in the presence and absence of wortmannin and PIK-III Cellular proteins were analyzed by IB for LC3-I, LC3-II, p62, and GAPDH using HRP-linked secondary antibody and ECL detection. Loading controls included GAPDH (cb1001mAb (6C5)). McA cells were incubated in 1\% BSA/DMEM with DMSO, wortmannin or PIK-III for $18 \mathrm{~h}$. Band intensities were measured using ChemiDocXRS+ system and evaluated using ImageLab 5.1 software. B, Measurement of AKT pathway as in Figure 4a with $(5,10$, and 20) and without insulin (0) in the presence and absence of PIK-III and wortmannin blotting for both AKT and PAKT with ratios depicted on the bottom. C, Measurement of total ApoB as in Figure 4a in both the media and infranatant in the presence and absence of PIK-III and wortmannin. D, Measurement of cellular Sortilin, LDLR (LS-C146979, 1:2000), PCSK9 (Ab125251, 1:2000) and GAPDH as in Figure 4a, in the presence and absence of PIKIII and wortmannin. E, Quantification of LDLR, Sortilin, and PCSK9 as in $4 a$. 
A

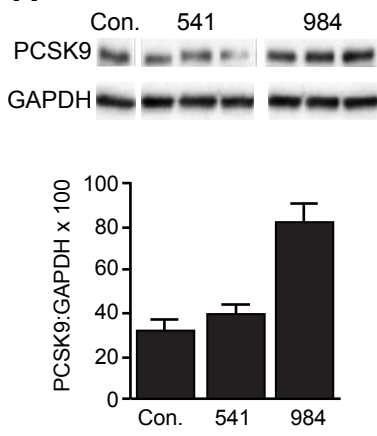

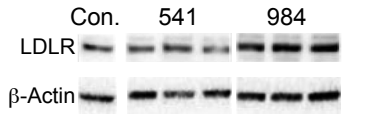

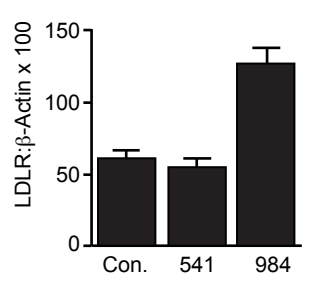

B

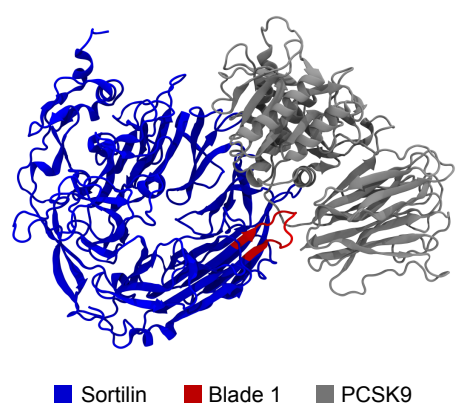

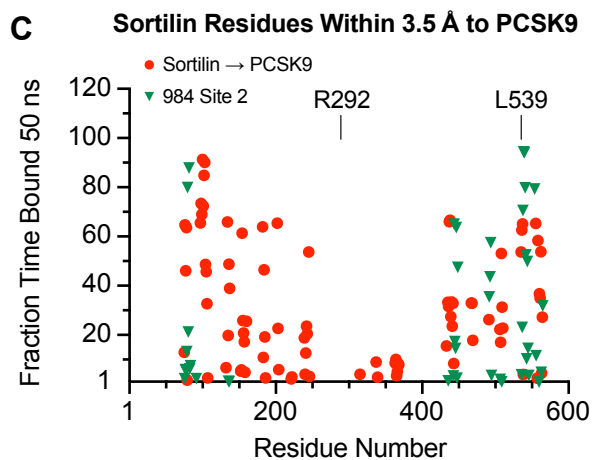
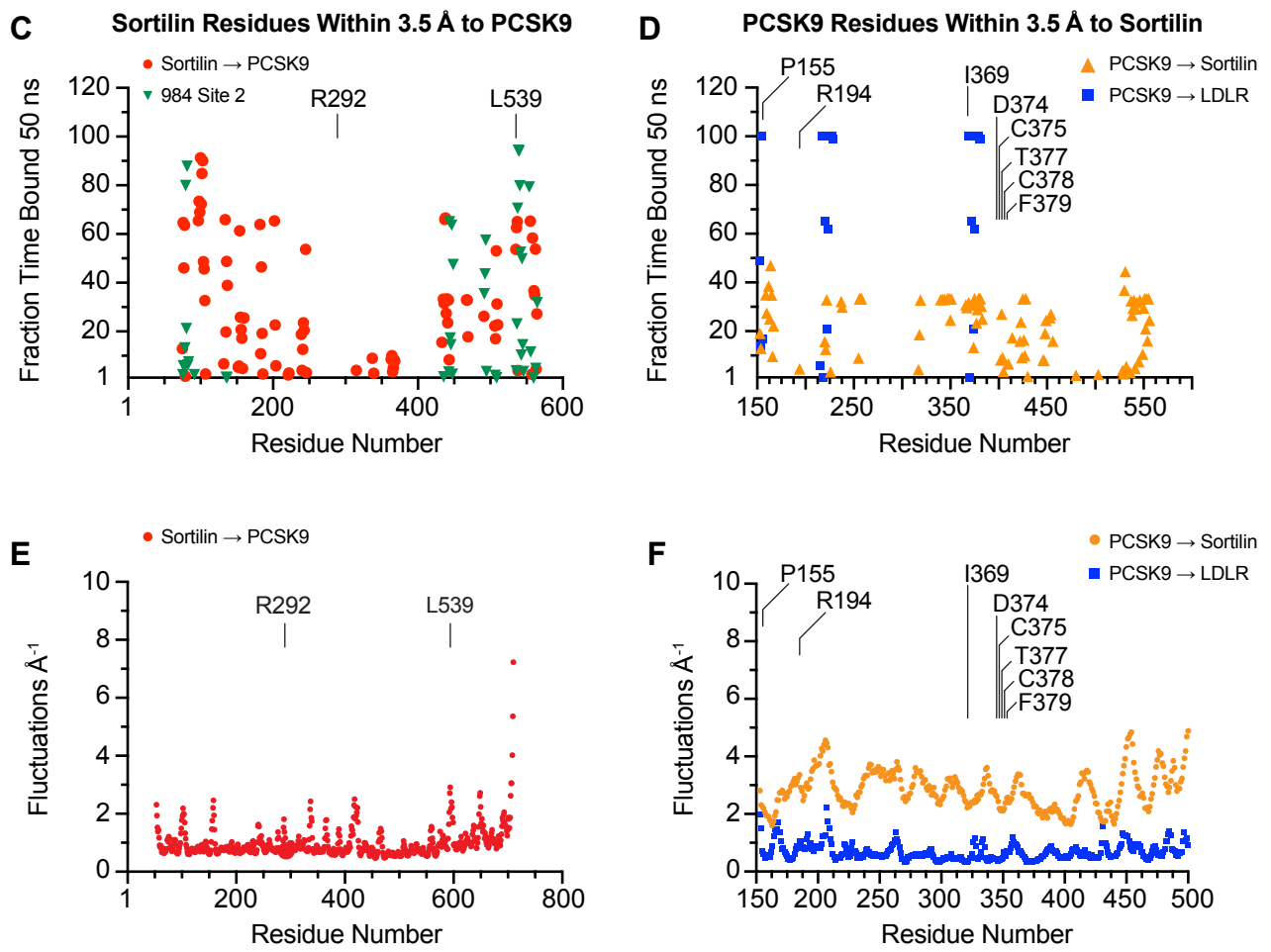

Figure 5. Effects of Cpd541 and Cpd984 on PCSK9/LDLR Expression and Potential Role of $\mathbf{P I}(\mathbf{3}, \mathbf{4}, \mathbf{5}) \mathbf{P}_{3}$ in Sortilin Binding to ApoB-Lp. A, McA cells were incubated in 1\% BSA/DMEM with DMSO, cpd541 $(10 \mu \mathrm{M})$ or cpd984 $(10 \mu \mathrm{M})$ for $18 \mathrm{~h}$. Cellular proteins were extracted and analyzed by IB for LDLR (LS-C146979, 1:2000) or PCSK9 (Ab125251, 1:2000) using HRP-linked secondary antibody and ECL detection. Loading controls included - $\beta$-Actin (Rockland ph600401-886) and GAPDH (cb1001mAb (6C5)). Band intensities were measured using ChemiDocXRS+ system and evaluated using ImageLab 5.1 software. B, Results from ClusPro protein-protein docking simulation of Sortilin PDB ID: 4PO7 to PCSK9 PDB ID: 2P4E with Sortilin 
in blue, PCSK9 in grey, blade 1 of Sortilin in red. C, Residue analysis as in Figure $2 \mathrm{e}$ corresponding to three 10 ns simulations of PCSK9 to Sortilin using PDB files in Figure 5b with mean fraction bound as in Figure 2e for PCSK9 to Sortilin with 984 results from Figure 2e plotted in green, PCSK9 residue. D, RMSF analysis of Sortilin residues from ClusPro simulation of Sortilin PDB ID: 4PO7 to PCSK9 PDB ID: 2P4E e, RMSF Analysis of PCSK9 residues from ClusPro simulation in Figure 5E. 

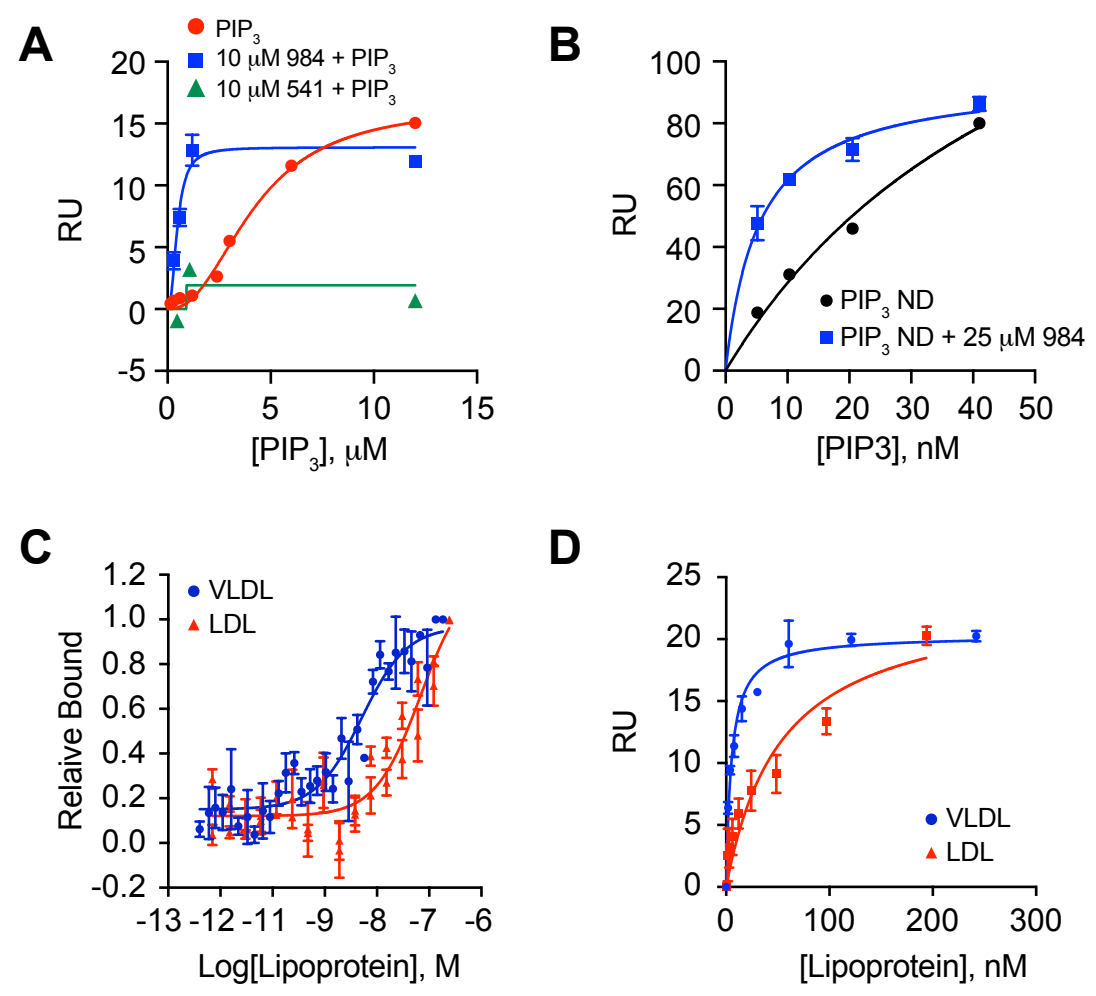

Figure 6. Cpd984 modulates binding to $\mathrm{PI}(3,4,5) \mathrm{P}_{3}$. A, SPR as in Figure 1 a of diC8-PI(3,4,5) $\mathrm{P}_{3}$ binding measurements in the presence and absence (red) of $10 \mu \mathrm{M}$ cpd984 (blue) and cpd541 (green) with $K_{D}$. B, SPR using a CM5 chip crosslinked with Sortilin. C, Labeled microscale thermophoresis (MST) using Atta-647 Ni-NTA labeled Sortilin measured in the red channel with a constant concentration of Sortilin of $12.5 \mathrm{nM}$ and 32 different concentrations of VLDL and LDL run in triplicate and analyzed using Nanotemper M.O. Affinity Analysis software with $K_{D}$ for VLDL of $\sim 4 \mathrm{nM}$ and for LDL of $\sim 74 \mathrm{nM}$. D, SPR performed using a CM5 chip containing $\sim 2000 \mathrm{RU}$ of Sortilin with $K_{D}$ for VLDL of $\sim 5 \mathrm{nM}$ and for LDL of $\sim 54 \mathrm{nM}$. 
A

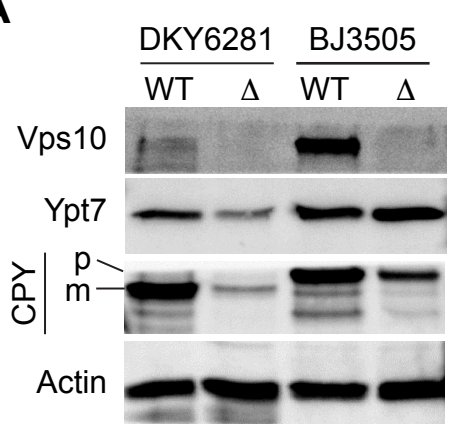

C

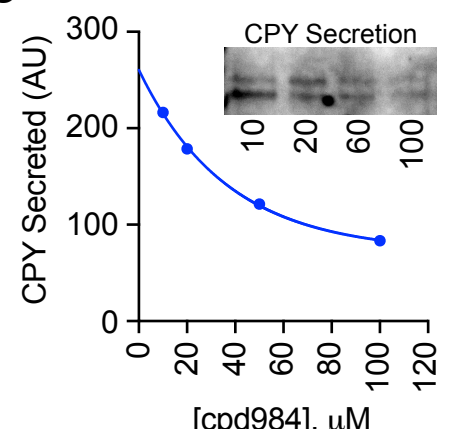

B

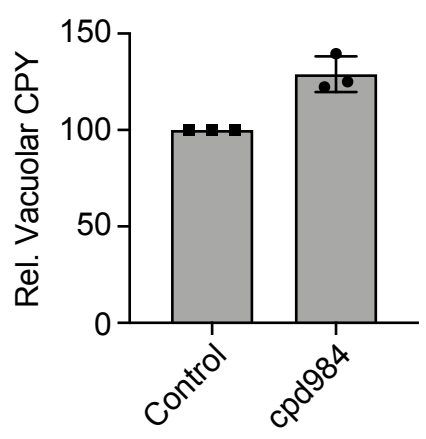

D

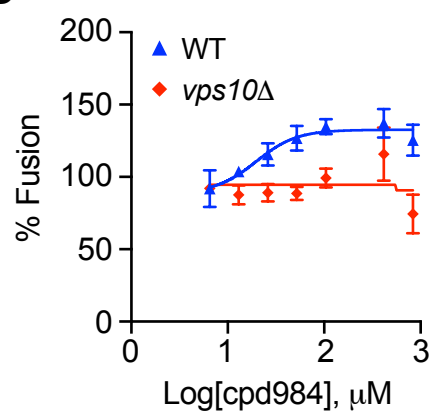

Figure 7. cpd984 affects CPY sorting in Yeast. A, Immunoblots of wild type and vps10 cell lysates with antibody against the late endosomal/lysosomal Rab Ypt7, the yeast Sortilin ortholog Vps10, the luminal protease CPY, and actin as a loading control. B, Relative concentrations of intracellular CPY in untreated WT cells vs cells treated with cpd984. C, Immunoblot of secreted CPY in cells treated with cpd984. D, In vitro homotypic vacuole fusion assays with wild type and vps10 vacuoles in the presence or absence of cpd984. 